\section{Preparation and Use of Needles and Micropipets for Handling Very Small Particles}

Anna Teetsov - McCrone Associates

To successfully isolate $1-100 \mu \mathrm{m}$ samples for microscopical examination or analysis, one needs a good set of microtools, with the most essential being the needle and the micropipet. This paper will describe how to make them and how they are used to solve various sample preparation problems.

\section{TUNGSTEN NEEDLES}

The three most commonly used needle types are the Fine, Medium and Curved. Less frequently used are Flat, Polyethylene and Eyelash needles. Relative sizes of the six needle types are shown in Figure 1.

A procedure for making tungsten needles has been carefully described in The Particle At/as, Edition Two, Volume I, by McCrone and Delly. The procedure is as follows:

24-gauge, $520 \mu \mathrm{m}$, tungsten wire is cut into one-inch lengths using wire nippers to minimize split ends because the wire is very brittle.

The tip of the wire is heated over a Meker burner or alcohol lamp until it is red hot; then it is quickly placed in $\mathrm{NaNO}_{2}$. The ensuing exothermic reaction is allowed to pro ceed for $1-5$ seconds. The end of a freshly cut tungsten wire may require 5 seconds to etch and form a sharp tip. One second may be sufficient to resharpen a damaged tip.

Over the past 20 years, minor changes have been made in the procedure described in The Particle At/as. A large number of needles may now be sharpened at one time. They are handled with tweezers and are not placed in a needle holder for sharpening.

The use of a sodium nitrite stick (as described in The Particle Atlas) is suitable for sharpening freshly cut tungsten wire. The relatively broad, unsharpened tip will stay hot long enough to put the glowing tip into the nitrate stick. This transfer of the needle from the flame to the $\mathrm{NaNO}_{2}$ stick must be done very quickly. If the tip is allowed to cool even slightly, the exothermic reaction will not be initiated.

For finer tips such as those needing resharpening, the required heat is lost too rapidly and they are bent when their cooled tips are pushed against the sodium nitrite stick. That is why the alternative method using the molten sodium nitrite is preferred for resharpening needles (see Figure 2).

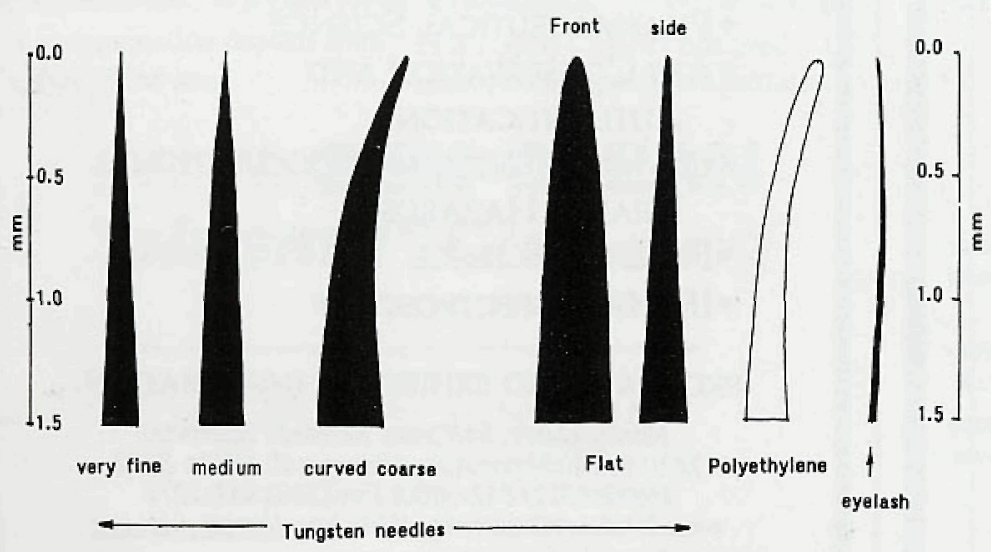

Figure 1: Relative sizes of the six needle types
TYPES OF TUNGSTEN NEEDLES

No special techniques are necessary for making Fine, Medium, Coarse or Flat needles. If a large quantity (more than 50) are made at one time, one will obtain approximately equal amounts of each type plus rejects. Rejects are needles with round tips, double tips (resulting from split wire at the tip), and needles with an uneven taper. These can be resharpened.

The freshly made needles are placed in a single pile in a petri dish lined with paper. Excess $\mathrm{NaNO}_{2}$ is removed by flowing a very fine stream of warm water into the petri dish for a few minutes. After the water is decanted, the needles are sorted into the five categories (Fine, Medium, Coarse, Flat and rejects). The good ones are immediately placed into flat clear plastic boxes with an elevated strip of adhesive tape to keep them in place (see Figure 3). The sharpened tips should be in a straight line so that one can compare the tips and make the proper choice of a needle for the task at hand.

A few of the coarse needles can be curved by applying pressure with a tungsten carbide scribe just above the tip as shown in Figure 4.

\section{POLYETHYLENE NEEDLES}

Polyethylene needles can be made from high density narrow polyethylene tubing. A $2-3$ inch piece of tubing is rotated and heated over an alcohol lamp and pulled out once it has softened. The pulling may have to be done in two or three stages to get a 5 $\mu \mathrm{m}$ tip within $2-3 \mathrm{~cm}$ (see Figure 5). These needles are very durable and a few of them can last up to a year of routine use.

\section{EYELASH NEEDLE}

A relatively straight Eyelash needle can be made by cutting 3 $\mathrm{mm}$ off the tip of an eyelash and attaching it to a medium tungsten needle tip with epoxy. The Eyelash needle can be cleaned by dipping the tip in ethanol or xylene. The needles are also stored, together with the tungsten needles, in clear plastic boxes (see Figure 3 ). above.

Table I lists the primary uses for the needles described

TABLE I

\section{NEEDLES FOR MICRO SAMPLE PREPARATION}

\begin{tabular}{ll}
\multicolumn{1}{c}{ Needle Type } & \multicolumn{1}{c}{ Primary Use } \\
Very fine tungsten & Manipulating particles $<20 \mu \mathrm{m}$ \\
Medium tungsten & Manipulating $>20 \mu \mathrm{m}-100 \mu \mathrm{m}$ \\
Coarse, curved tungsten & Manipulating drops of solvents \\
Flat tungsten & Scraping fine residues off \\
Polyethylene & $\begin{array}{l}\text { Performing aqueous extractions } \\
\text { Eyelash }\end{array}$ \\
& $\begin{array}{l}\text { Manipulating samples on very fragile } \\
\text { surfaces }\end{array}$
\end{tabular}

Continued on page 10

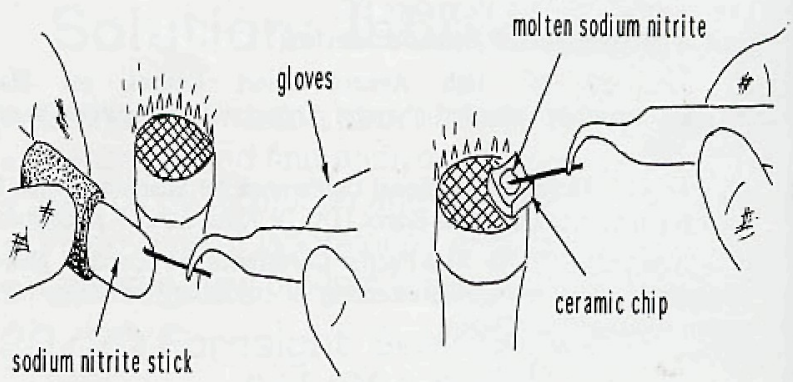

Figure 2: Two ways to sharpen tungsten wire 


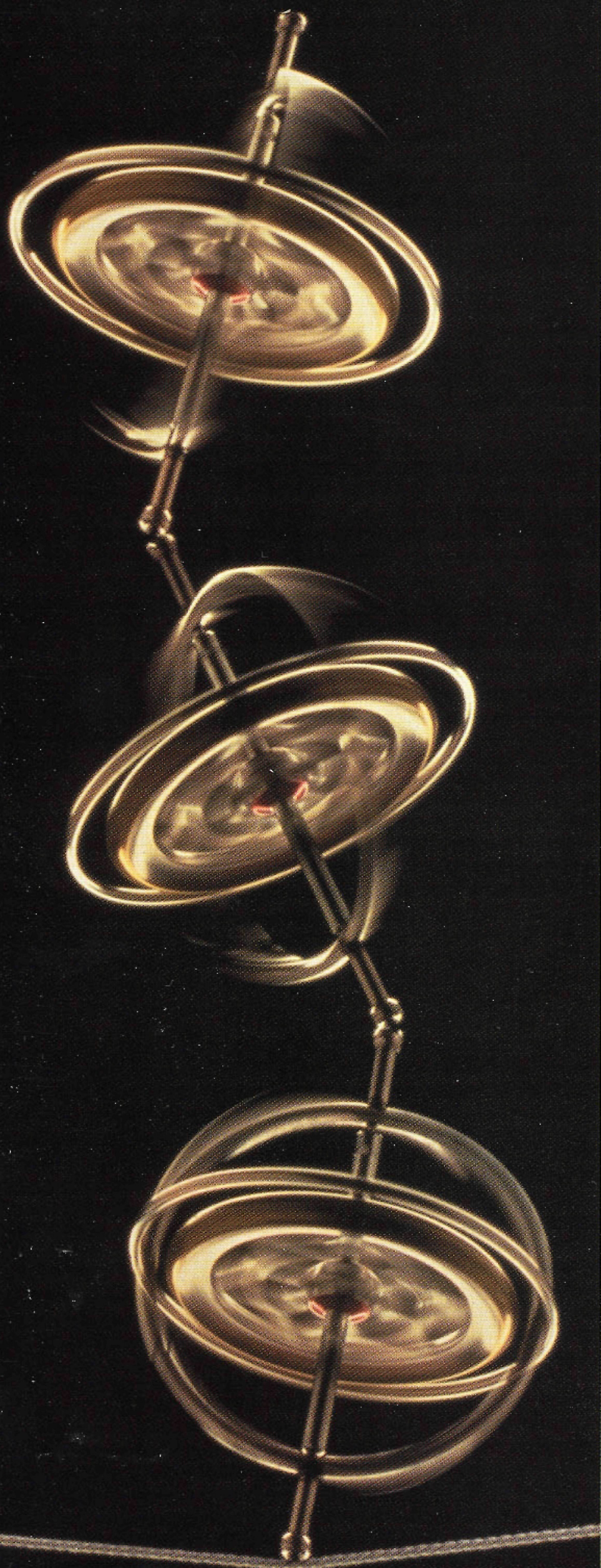

\section{Getting All Your Detectors} Working Together Can Be A Tough Balancing Act.

\section{That's Where Emispec Comes In.}

If balancing all components of your detectors has your head spinning, you should be talking to us.

You see, at Emispec Systems, Inc., we approach data acquisition differently. Instead of creating systems targeting one detector, we focus on integration. This concept can be applied equally to new and existing electron microscope installations. Core acquisition capabilities of our products include:

- Digital scanning for STEM.

- Digital EDX acquisition and analysis.

- EELS acquisition and analysis.

- $\mathbf{C C D}$ and TV imaging.

Integrated microscope control, imaging and spectroscopy allows automation of demanding experiments, such as spectrum imaging. Emispec enhances these capabilities with extensive on-line and off-line processing.

To find out how Emispec can help your lab keep in balance, visit our Web site today at www.emispec.com. See why we are fast becoming the leader in microscope detector technology solutions.

\section{Emispec}

Emispec Systems, Inc

2409 South Rural Road, Suite D

Tempe, Arizona 85282 USA

Phone: 480.894 .6443 - Fax: 480.894 .6458

Web: wnw.emispec.com

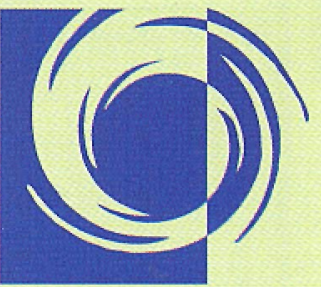

thinking beyond the box ${ }^{\mathrm{TM}}$ 
Preparation and Use of Needles and Micropipets for Handling Very Small Particles

Continued from page 8

\section{NEEDLE HOLDERS}

The 24-gauge tungsten wire will fit most needle holders. The aluminum needle holders shown in Figure 6 are preferred because they are light and have just the right length. A number of them should be on hand, one for each of the various types of needles.

Since polyethylene needles do not fit into these holders, it is helpful to fit them with colorful micro vacuum cups (obtained from an auto supply store) so they can be readily located on the microscope bench (see Figure 7).

\section{TUNGSTEN NEEDLE CLEANING}

Before a fresh tungsten needle is used, it should be cleaned thoroughly by passing it a number of times through a needle cleaner made from a sheet of cleanroom paper held by the lid of a small canning jar (see Figure 8). The paper is wetted and the soft, wet paper cleans the needles very well as they are passed through. Dry paper does not clean as efficiently and may damage a fine tip. By putting a drop of amyl acetate on the paper, one can remove excess collodion from contaminated needles by passing the tips a number of times through the solvent-treated paper fibers.

\section{POLYETHYLENE MICROPIPETS}

Polyethylene micropipets are essential for most sample preparation techniques. Because they are very small, they are only used with the stereomicroscope. They deliver small drops of solvent by capillary action and are filled by capillary action as well. They can be made from high density polyethylene tubing by heating the tubing in stages. The procedure is similar to that used in making the polyethylene needles, except the tubing is not fused and the tip is trimmed so that it will deliver a drop of
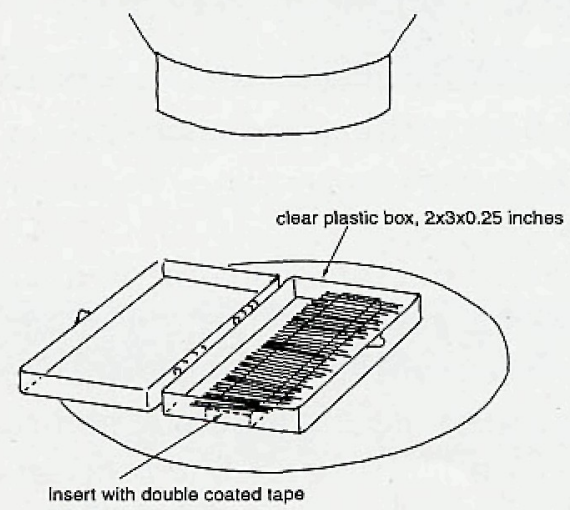

Figure 3: Proper storage of sharpened tungsten needles

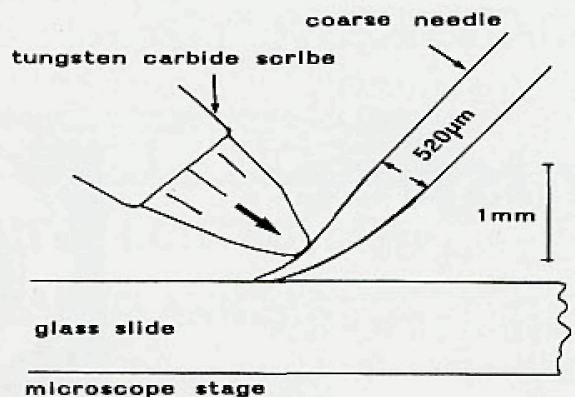

Figure 4: Making a curved needle for manipulating solvents. solvent every time it is touched to a surface (see Figures 5 and 9).

These micropipets can also be made from 1-10 $\mu \mathrm{L}$ pipetter tips made of low density polyethylene. These pipets are not as durable as the ones made from high density tubing, but they are much easier to make (see Figure 9 ) and fit well into our solvent dispensers described below.

The micropipets are designed to be used under a stereomicroscope. They are small and obscure little of the field-of-view. When properly held, the tip of the micropipet will remain in focus. They reliably deliver a small drop of solvent when the tip is touched to a glass slide (see Figure 10). The size of the drop depends on the size of the tip and the amount of liquid in the pipet. The size of the drop can also be controlled by the position of the tungsten needle. As the needle is lowered, it will pull more solvent from the micropipet.

The micropipet can be filled from a $15 \mathrm{~mL}$ ground glass bottle Continued on page 12

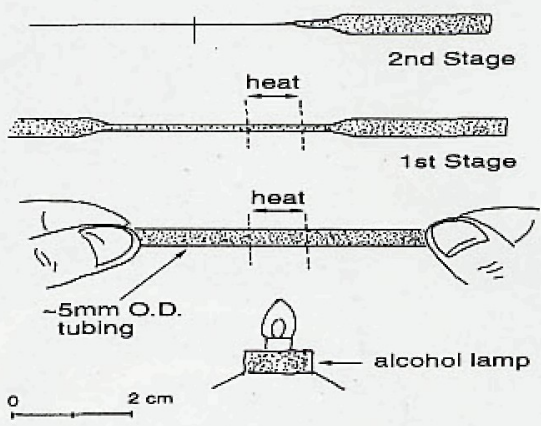

Figure 5: Making a polythylene needle.
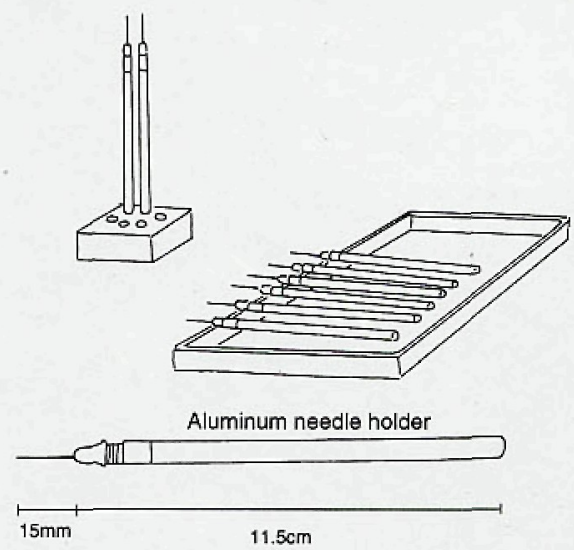

Figure 6: Needle holder, storage tray, and lucite holder.

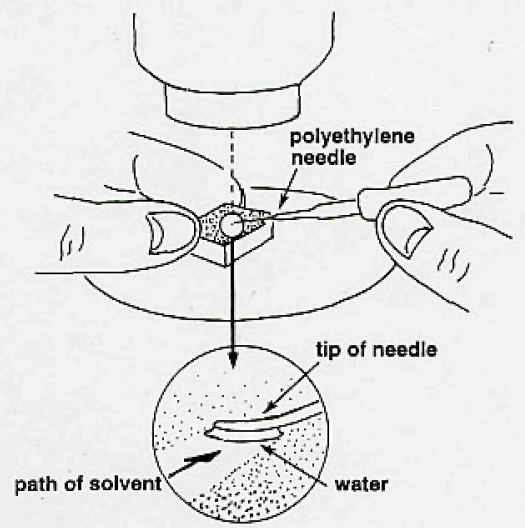

Figure 7: Extracting a water soluble residue with a polyethylene needle 

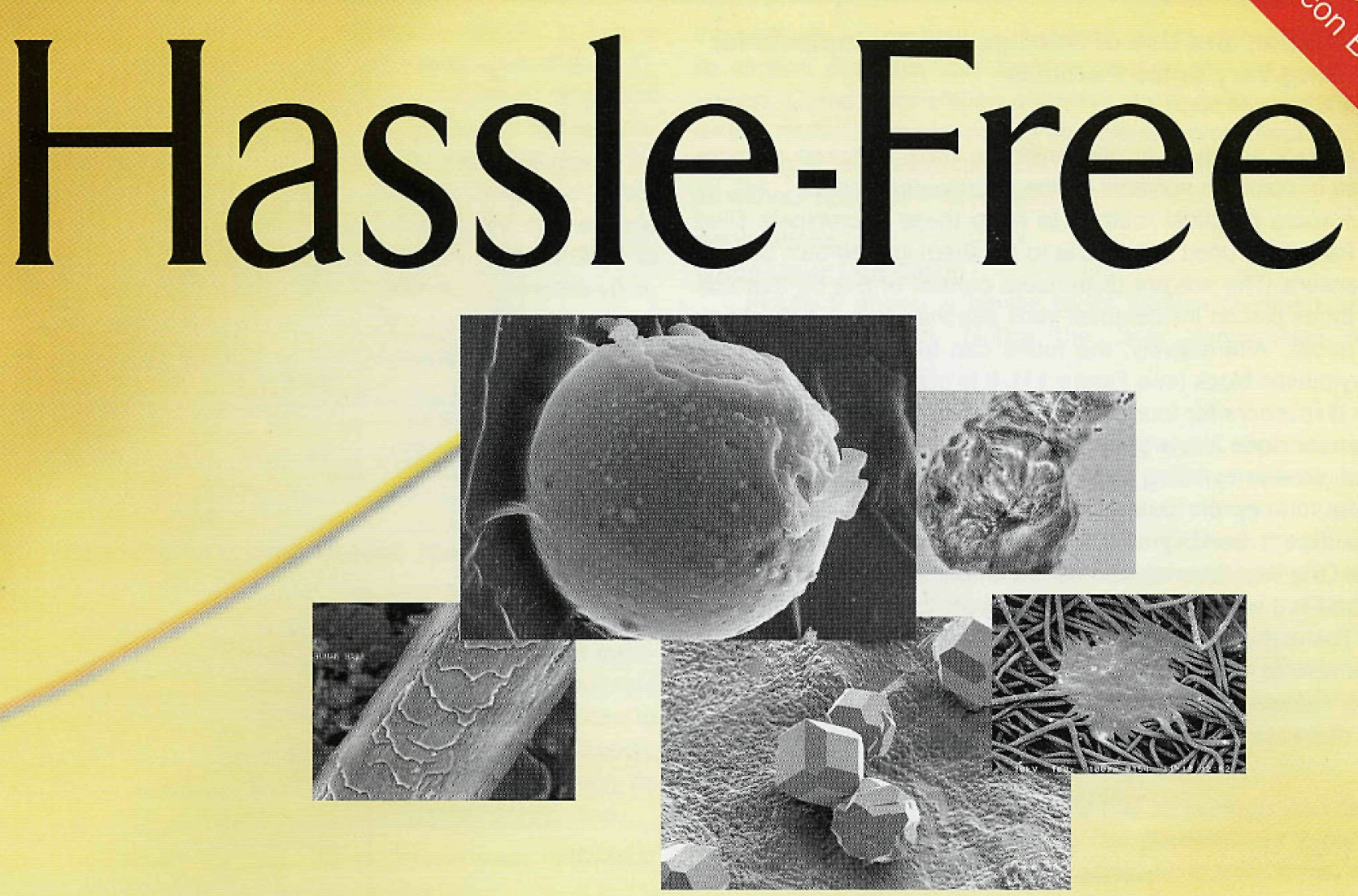

\section{Automated Microanalysis and Microscopy....Just a Click Away}

Announcing the

Personal Automated Feature Analysis System

Looking for comprehensive, no hassle, unattended SEM/EDX analysis. Take a look at the unique

Personal Automated Feature Analysis System (PAFA). A sophisticated software package that enhances the Personal SEM into a highly sophisticated, automated microanalyzer .

\section{Automation for whatever your application}

We say

"Personal" because

PAFA can be truly customized for a broad spectrum of specific applications. Such as gun shot residue, wear debris, metal inclusion to mention a few. The list is long. Whatever you want analyzed. Particles, features, inclusions, defects and much more, can be done quickly and thoroughly. If you need real time, "live" detection, it's part of the package. The best part. You, personally, no longer have to spend all that time to diligently find all those particles. Customized reports are given with all the critical information you need. All easily, unattended and with no hassles.

\section{No company does microanalysis automation better}

Why do we do it better? It's simple. Every component, the SEM, EDX, stage control, the software, is from one source - R J Lee Instruments, a microanalysis expertise that is unsurpassed. No integrating problems, no finger pointing from one manufacturer to another, and yes, no hassles. If there ever is a problem, you have a single source supplier and guarantee-R J Lee Instruments.

Take a closer look at hassle-free automation today. Call 1-800 538-6850 or visit our website www.rjleeinst.com. 


\section{Preparation and Use of Needles and Micropipets for Handling Very Small Particles \\ Continued from page 10}

by capillary action. It is convenient to have a dozen or more bottles of common solvents on the microscope bench.

A more practical method to keep these micropipets filled with frequently used solvents is to put them in their own solvent dispensers. The solvent dispensers consist of $6 \times 50 \mathrm{~mm}$ culture tubes placed inside small vials attached to a 1 inch aluminum block. Alternatively, the tubes can be put directly into a heavy plastic block (see Figure 11). It is preferable to have four such dispensers for four micropipets. Keep amyl acetate in one dispenser since it is a good solvent for thin films of flexible collodion used in handling small particles. Nonane is also a good solvent to keep on hand. It does not evaporate quickly, allowing sufficient working time for doing extractions or manipulations. One dispenser should be left empty so that its micropipet can be filled with the desired solvent from a $15 \mathrm{~mL}$ bottle.

The last dispenser contains $n=1.662$ oil. It is convenient to be able to dispense $1 \mathrm{~mm}$ drops of the oil when examining micro samples under a $1-3 \mathrm{~mm}$ coverglass. The micropipets and dispensers can be color-coded using the micro vacuum

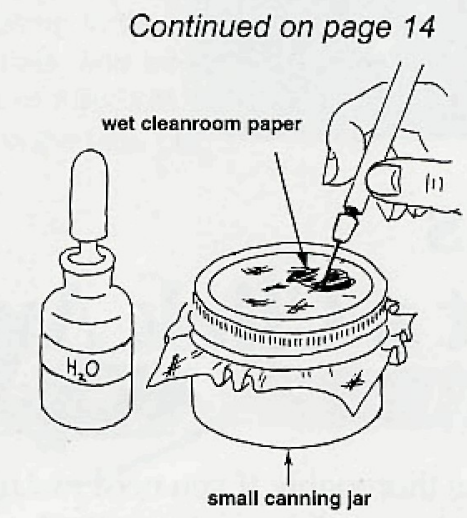

Figure 8: A needle cleaner

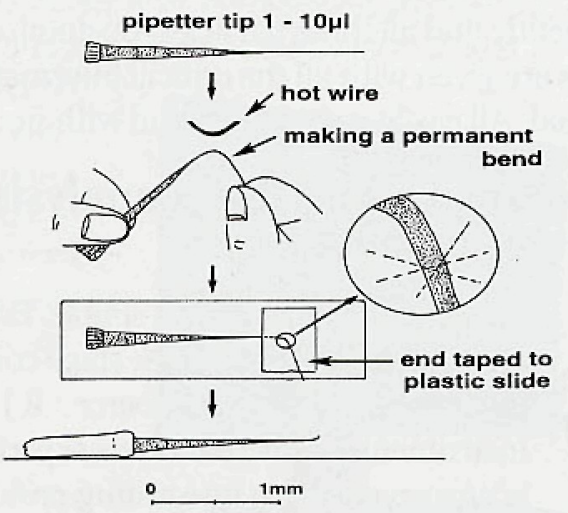

Figure 9: Micropipets from 1-10 $\mu \mathrm{L}$ pipet tips.

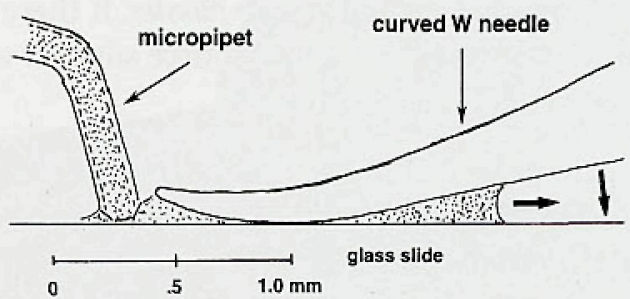

Figure 10: Increasing the amount of solvent delivered by a micropipet. As the needle is lowered more solvent will flow beneath it.

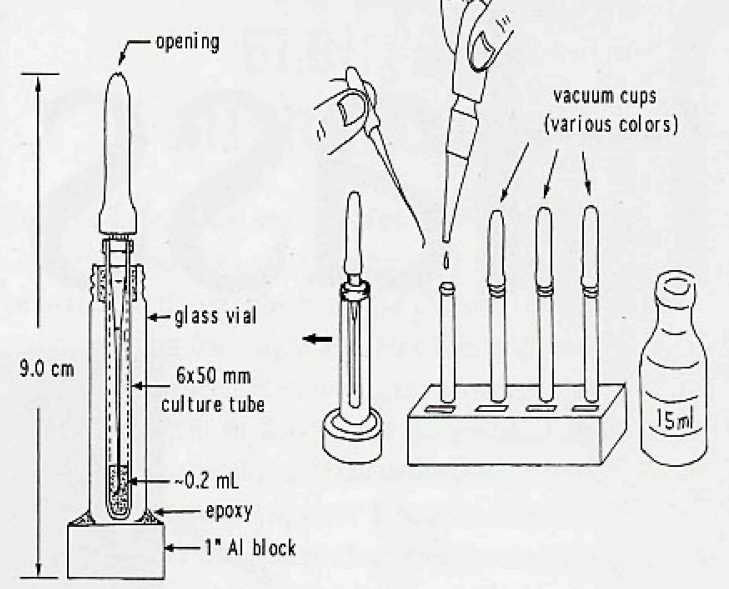

Figure 11: Solvent dispersers for micropipets.

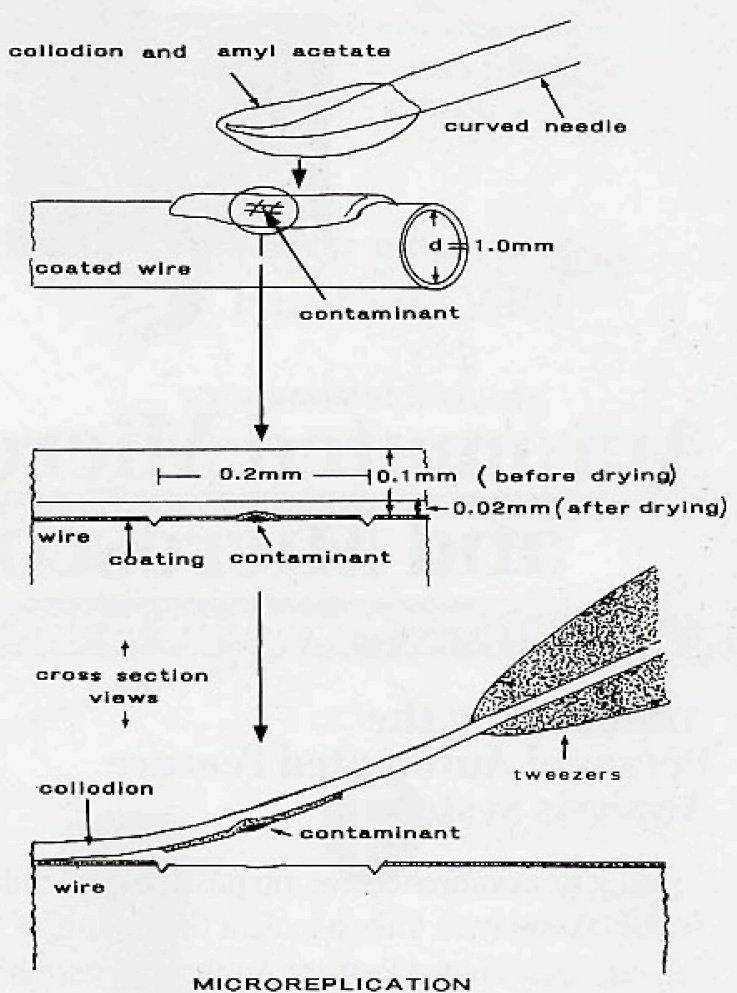

Figure 12: Removing a small defect with a collodion film.

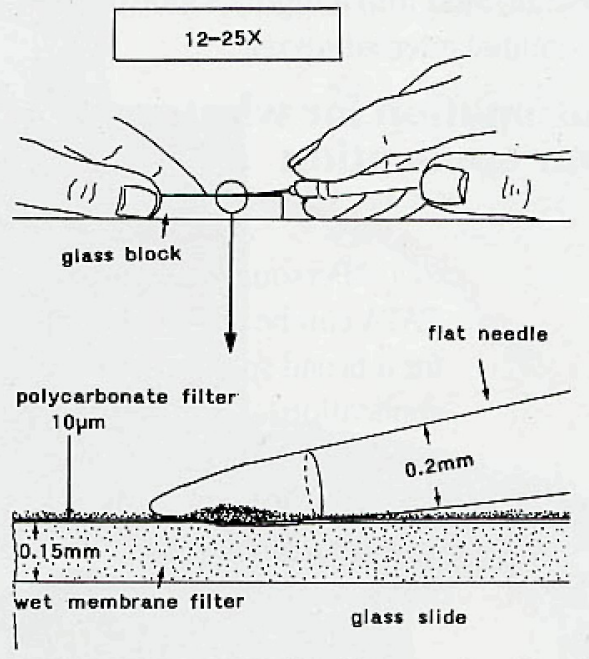

Figure 13: Collecting nanogram residue from a polycarbonate filter with a flat tungsten needle. 


\section{$\gamma \mathbf{P} \mid \mathbf{G} \mathbf{T}$

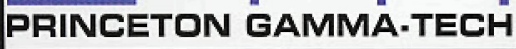
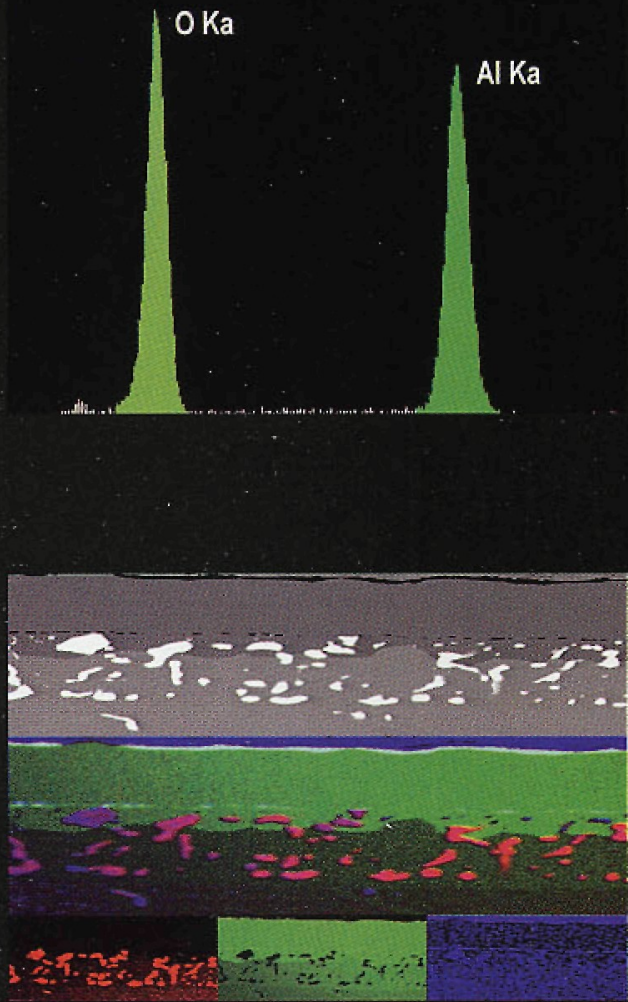

\section{Avalon 4000:}

\section{Low cost EDS upgrade!}

\section{Avalon 8000:}

\section{Workhorse for}

\section{everyday solutions!}

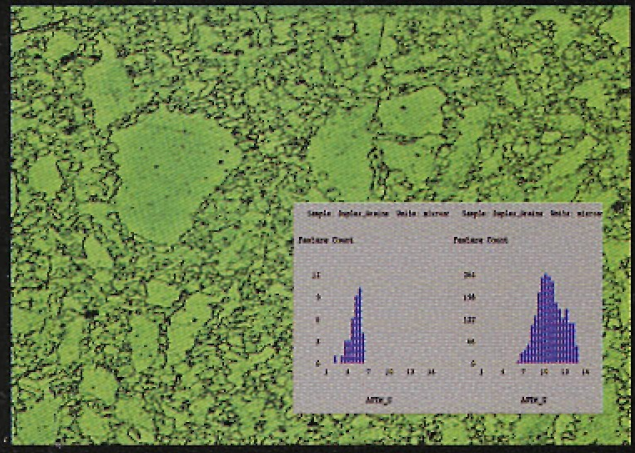

\section{IMIX:}

\section{Simply the best!}

For over 30 years you have come to PGT for the latest innovations in X-ray microanalysis and EDS detector technology.

Princeton Gamma-Tech, Inc. C/N 863 Princeton, NJ 08542-0863

Telephone: (609) 924-7310

Facsimile: (609) 924-1729

e-mail: sales@pgt.com
Now you can come to us for inexpensive upgrades, fundamental workhorse systems, as well as solutions for advanced applications. 


\section{Preparation and Use of Needles and Micropipets for Handling Very Small Particles \\ Continued from page 12}

and dispensers can be color-coded using the micro vacuum caps.

The advantage of having these dispensers is that they keep the micropipets filled with solvent all the time and only one hand is required to pick up the pipet. To fill a micropipet from a $15 \mathrm{~mL}$ ground glass bottle requires two hands. When manipulating small samples, one may only have one hand free.

Another advantage of these dispensers is that they emit less organic vapors than a $15 \mathrm{~mL}$ ground glass bottle that is opened and closed throughout the day. Note that less than one-quarter of the glass vial is filled with solvent. Surprisingly, that amount of amyl acetate or nonane will take 2-3 days to evaporate.

\section{A FEW COMMON USES}

FOR THE VARIOUS TYPES OF NEEDLES

Very Fine tungsten needles are used to pick up a loosely held, 1-10 $\mu \mathrm{m}$ particle directly from a surface and deposit it on a substrate for further analysis. These needles are usually used only once because the tip is frequently damaged in the process.

Medium needles can be used to pick up larger particles, either directly or with soluble gum. Because of their greater strength, these needles are used most frequently. However, they quickly develop slight imperfections which are hard to observe. Since these damaged needles may not release particles properly, they should be changed often, even though they may look undamaged.

The Curved needle is used mainly to manipulate $1 \mathrm{~mm}$ drops of solvent on substrates because it can hold a large volume of solvent beneath it due to its large diameter and curved tip. Also, it is used to transfer embedding media for micro replication (see Figure 12).

The Flat needle is ideal for removing fine precipitates from smooth, soft polycarbonate filters. Since the needle has no sharp tip and will not scratch a surface, it can be used like a

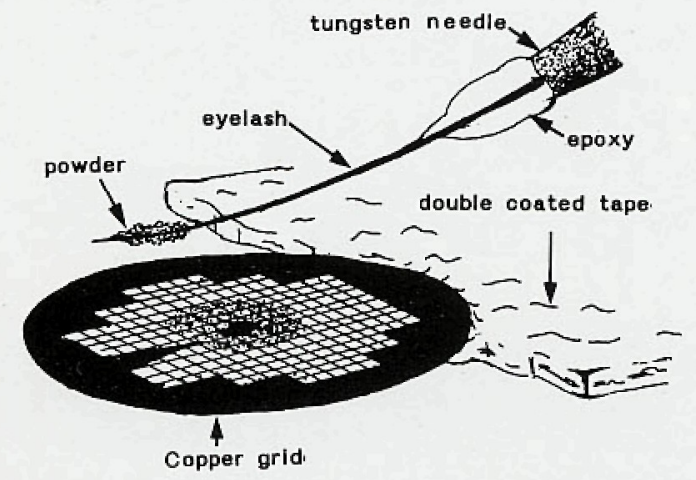

Figure 14: Dispersing a powder on a copper grid with an eyelash needle.

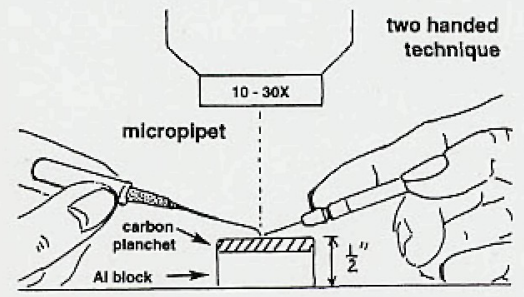

Figure 15: Preparing a sample for electron microprobe analysis. spatula or a knife. It is very sturdy and can be reused many times (see Figure 13).

The Eyelash needle is used to disperse, without using any solvents, fine powders on a carbon-coated copper grid for analysis by transmission electron microscopy (see figure 14). It requires little skill and gives very nice results. The eyelash needle, unlike the tungsten needle, is not strong enough to break the thin carbon film on the grid. The needle can be rinsed and reused many times.

\section{SOME APPLICATIONS OF THE MICROPIPET AND NEEDLES}

The micropipet is not used by itself; it is always used with the needle. This is referred to as the "two-handed technique" (see Figure 15). The two are used together to tack small samples on carbon or beryllium surfaces for further analysis. Frequently, a drop of solvent is all that is necessary to keep a fine powder or a few flakes in place. Larger samples may require a little collodion or soluble gum to hold them down.

Small drops of solvent may help remove a small particle or a fine powder off the tip of a tungsten needle. Groups of small particles can be concentrated or dispersed in small, $1 \mathrm{~mm}$, drops of solvent for further analysis.

Small drops of solvent are used to make approximate solubility checks on nanogram sized samples as shown in Figure 16. The edge of the drop is moved back and forth over the particles, dissolving those that are soluble and leaving the others in place. Only the edges of the drop should be used, because the center portion of the drop beneath the tungsten needle has too much turbulence and may dislodge the particles from the glass slide. This makes it difficult to tell if the particles have dissolved or simply moved out of the field-of-view.

The needle and micropipet are ideal for doing micro extraction. Extraction of oil from a small particle serves as an example. A small particle can be placed on a $4 \times 5 \mathrm{~mm} \mathrm{KBr}$ crystal and, with

\section{Continued on page 16}

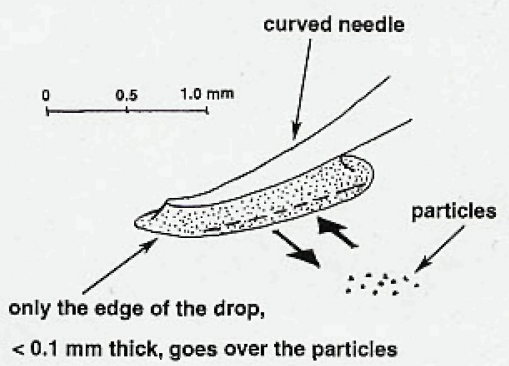

Figure 16: Checking the solubility of nanogram samples.

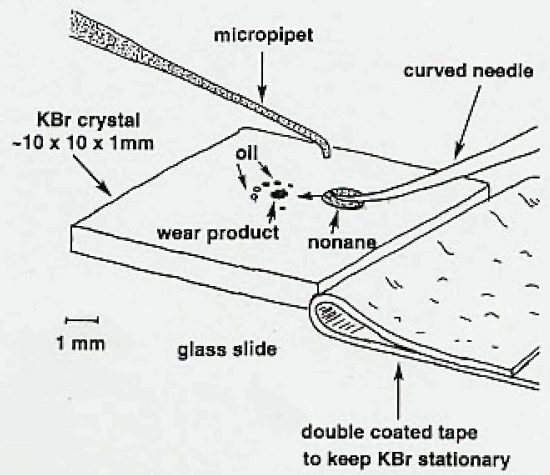

Figure 17: Extracting a noname soluble fraction from a wear product. 


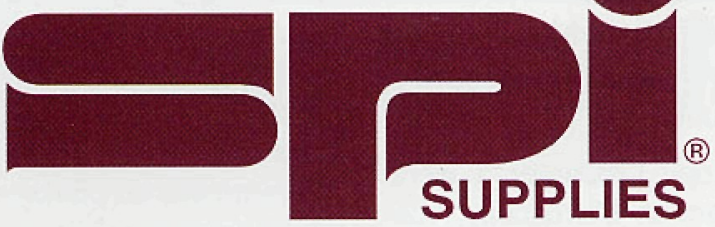

E File Edit Diew Go Bookmarks options Directory Help

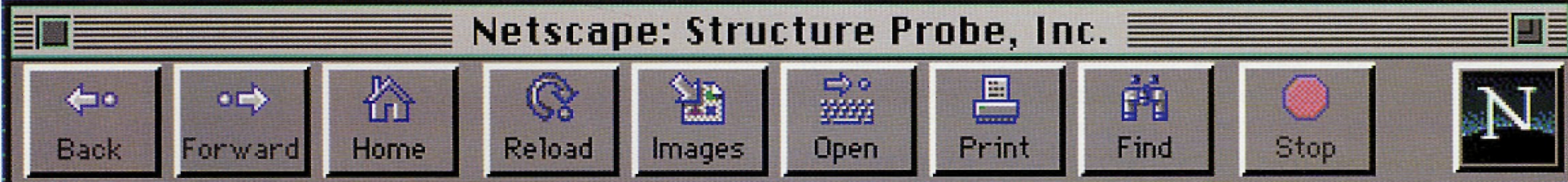

\section{What's New? What's Cool? Handbook Net Search Net Direstory Newsgroups}

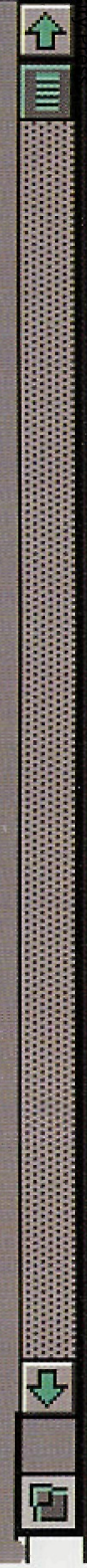

SPI Supplies Division of STRUCTURE PROBE, Inc.

P.O. Box 656 - West Chester, PA 19381-0656 USA

Ph.: 1-610-436-5400 • 1-800-2424-SPI (U.S. only) • FAX: 1-610-436-5755 • E-mail: spi2spi@2spi.com 
Improving the Resolution of Sputter-coated Films

Mary Mager, University of British Columbia mager@interchange.ubc.ca

After an inquiry from the Microscopy Listserver, I went back to my 1980 copy of Scanning Electron Microscopy, volume I. Several authors had investigated the structure of thin metal films by depositing the films onto carbon-film-covered TEM grids and imaging the films at high magnification. There were several proposals for new devices that have since become standards for high-resolution coaters ${ }^{1,2}$, but the Listserver inquiry was for a fine conducting film suitable for high-resolution SEM from an existing sputter coater ${ }^{3}$. There were several factors studied that influenced the fine structure of the films. The first was the materials sputtered: for a given set of conditions of voltage, current and time, platinum gave the finest film, $60 \%$ gold $-40 \%$ palladium ( $\mathrm{Au} / \mathrm{Pd}$ ) the next finest and pure gold the least fine. Other materials such as tungsten ${ }^{4}$, tantalum, nickel, chromium and molybdenum were also tried and gave very fine films with almost undetectable structure, but they will all oxidize, so they are not good for long-term storage of specimens after sputtering. I tried a nickel target in my coater, coated a carbon-covered grid and examined the resultant film in the TEM. The film was thin and the structure was almost undetectable, but I never used it on SEM specimens, so I don't know how well it coated the specimens for practical use.

The second factor that influenced the sputtered structure was the temperature of the substrate and sample as they were being sputtered ${ }^{3}$. Lowering the temperature seems to reduce the mobility of the metal atoms after they hit the sample surface, preventing them from agglomerating into large clumps and keeping the film small-grained and thin. Some sputter coaters have sample cooling capabilities and I have heard of someone making a sample holder block, insulated with teflon and grounded with a wire, which was chilled in a freezer or liquid nitrogen before being put in the coater. You must let it warm up completely after coating and before venting the sputter coater, to prevent moisture condensation, but it does make the coating finer-grained if all the other parameters remain constant. A third factor was accelerating voltage. Some sputter coaters allow you to adjust the voltage and I found that I could lower the voltage on my unit to $700 \mathrm{~V}$ and $25 \mathrm{~mA}$ current for three minutes to sputter $\mathrm{Au} / \mathrm{Pd}$ and yield a fine enough film for my use. If I used pure gold I could lower the current to $20 \mathrm{~mA}$. The argon pressure was maintained at 150 to 200 millitorr.

While doing experiments on the imaging of charge in a ceramic sample that sintered together a conductive $(\mathrm{SiC})$ phase with a non-conductive phase $\left(\mathrm{Al}_{2} \mathrm{O}_{3}\right)$, I tried to do a very short (ten second) coating, under my usual conditions of $700 \mathrm{~V}$ and $25 \mathrm{~mA}$, to retain a bit of charging to differentiate the phases. To my surprise this rendered the surface completely conductive. I found it worthwhile, particularly with smooth samples, to try very short coatings and re-coat again if the first was not quite enough. This is very dependent on the surface area of the sample, but the film has much less structure when it just starts to nucleate than after prolonged bombardment has heated the substrate and re-crystallized the thin film. I have found that with difficult samples, such as fluffy bugs, it is better to do several short coats, turning the sample between each coat, and to use some conductive paint to connect the area of interest to the sample stub, so the coating does not have to conduct too far. Tilting the sample on its side for a second coat is useful to get the coating under edges or to connect round particles to the substrate. See following illustration.

Since I do not have a field-emission SEM, I cannot evaluate the structure of the finest coatings; so these measures are enough to provide featureless coating for my use. Imaging a goldsputtered film on polished graphite is actually a good way to check a high resolution SEM for astigmatism and sharpness.

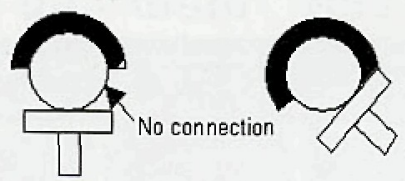

first coat second coat

Coating a second time with the sample at a 45 degree tilt may help complete the path to ground

1. Peters, K. R. 1980. Penning Sputtering of Thin Metal Films for High Resolution Electron microscopy. SEM/1980/l, SEM Inc., AMF O'Hare, IL. 143-154.

2. Franks, J., Clay, C. S., Pease, G. W. 1980. Ion Beam Thin Film Deposition. SEM/1980/I, SEM Inc., AMF O'Hare, IL. 155-162.

3. Echlin, P., Broers, A. N., Gee, W. 1980 Improved Resolution of Sputter-coated Metal Films. SEM/1980/l, SEM Inc., AMF O'Hare, IL. 163-170.

4. Slayter, H. S. 1980. High Resolution Metal Coating of Biopolymers. SEM/1980/I, SEM Inc., AMF O'Hare, IL. 171-182.

\section{Preparation and Use of Needles and Micropipets for Handling Very Small Particles \\ Continued from page 12}

small drops of nonane carefully guided with a needle over the particles, any oil in them can be extracted right on the crystal for IR analysis (see Figure 17). The reason that this extraction works is because the whole drop deposited by the micropipet, as well as the particle, is in the field-of-view at 10 or $20 \mathrm{X}$. One can watch the drop going to dryness and one is able to observe a small amount of oily residue after the nonane evaporates. One can immediately mark the position of the residue with a tungsten needle and run a blank next to the residue by placing a drop of pure solvent to check for a deposit. Large drops, such as those taken directly from the $15 \mathrm{~mL}$ ground glass bottles, would not work on the small $\mathrm{KBr}$ crystal or any other surface if the sample is 100 times smaller than the drop. Also, it would be difficult to keep the large drop together as it goes to dryness.

Frequently, a tiny piece of polyester filter may be used to remove a micro drop of oil from a hard-to-reach place. To extract the oil from this filter for further analysis, a small drop of nonane is deposited on the surface of the $\mathrm{KBr}$ and immediately the filter, held on the tip of a needle, is dipped in the solvent. Most of the oil will remain with the solvent and, as the solvent evaporates, one will see an oily drop appear on the surface of the crystal. The position of the drop is marked with the needle because small drops are hard to relocate once the field-ofview is changed.

These are just some of the ways that tungsten needles and micropipets have been used. There are many more.

Anna Teetsov joined McCrone Associates in 1961. Though busy as a Senior Research Microscopist, she also teaches microsample preparation as part of the microscopy course curriculum offered at McCrone Research Institute.

Anna is this years recipient of the Chamot Medal awarded by the State Microscopical Society of Hinois. This annual award, recognizing an individual who has made outstanding contributions to the field of microscopy, will be presented at the INTER/MICRO-2000 conference this June. And if you would like to observe her photomicrography talents, take a look at the "Polypropylene with Phthalocyanine Blue Pigment" photomicrograph on the Nikon 2000 calendar. 